\title{
Unilateral destruction of the medial agranular cortical projection zone in the dorsocentral striatum produces severe neglect in rats
}

\author{
THOMAS M. VAN VLEET, KAREN J. BURCHAM, and JAMES V. CORWIN \\ Northern Illinois University, DeKalb, Illinois \\ and \\ ROGER L. REEP \\ University of Florida, Gainesville, Florida
}

\begin{abstract}
Physiological studies have indicated that neglect and recovery from neglect induced by unilateral lesions of the medial agranular cortex (AGm) in rats is correlated with reductions of metabolic activity and plastic changes in glutamate receptors in the dorsolateral quadrant of the striatum. The dorsolateral quadrant of the striatum encompasses the dorsocentral striatum (DCS), the projection zone of AGm efferents. Although these changes in function in the dorsolateral striatum are correlated with neglect and behavioral recovery, direct evidence for the role of this region is lacking. In the present study, we examined the behavioral effects of unilateral destruction of the DCS on neglect, as assessed by orientation to visual, auditory, and tactile stimulation. Subjects received a unilateral $N$-methyl-D-aspartate lesion of the DCS, a control lesion in a laterally adjacent site in the striatum, or an injection of vehicle into the DCS. The subjects were tested for neglect three times per week for 3 weeks by rating the magnitude of orientation to visual, tactile, and auditory stimulation. The results indicate that the DCS group demonstrated significantly more severe neglect than did the lesion control group or the vehicle controls. The results suggest that the DCS is part of a system for appropriate orientation to sensory stimuli in rats and that the DCS may play a pivotal role in behavioral recovery from neglect produced by AGm lesions.
\end{abstract}

The neglect syndrome is a complex and devastating disorder characterized by spatial and attentional deficits and by unresponsiveness to the side of space opposite a brain lesion (Heilman, Watson, \& Valenstein, 1993). In humans, most cases of neglect result from cortical damage in the right hemisphere to the inferior parietal lobule, the dorsolateral prefrontal cortex (frontal eye field), or the cingulate cortex. Although found less frequently, damage to the basal ganglia, the white matter of the internal capsule, and the mesencephalic reticular formation have also been reported to produce neglect (Heilman et al., 1993; Mesulam, 1990; Vallar \& Perani, 1986). The results of these studies have led to the hypothesis that it is likely that neglect is due to the disruption of a specific corticosubcortical network that is involved in directed orientation to sensory stimulation (Corwin \& Reep, 1998; Heilman et al., 1993; Mesulam, 1990).

A rodent cortical model of neglect has been developed to study the mechanisms of neglect and behavioral recovery from neglect following cortical lesions (Crowne \& Pathria, 1982; Crowne, Richardson, \& Ward, 1983). In the

Correspondence concerning this article should be addressed to J. V. Corwin, Department of Psychology, Northern Illinois University, DeKalb, IL60115 (e-mail: tj0jvcl@corn.cso.niu.edu). rat cortical model, neglect is primarily associated with unilateral lesions of the medial agranular cortex (AGm; Corwin et al., 1986; King \& Corwin, 1992, 1993; Reep, Corwin, Hashimoto, \& Watson, 1984, 1987; Reep, Goodwin, \& Corwin, 1990), the posterior parietal cortex (PPC; Chandler, King, Corwin, \& Reep, 1992; King \& Corwin, 1992, 1993; Reep, Chandler, \& Corwin, 1994), or the ventral lateral orbital cortex (VLO; King, Corwin, \& Reep, 1989). Unilateral destruction of the AGm, the PPC, the VLO, or the connections between the AGm and the PPC results in severe neglect of visual, auditory, and tactile stimulation and in allesthesia/allokinesia (orientations to the side opposite the stimulation; Burcham, Corwin, Stoll, \& Reep, 1997; Corwin et al., 1986; Crowne \& Pathria, 1982; Crowne et al., 1983; King \& Corwin, 1990, 1992, 1993; King et al., 1989).

Most of the research in the rodent model of recovery from neglect induced by cortical lesions has focused on the AGm, because of the close correspondence between these findings and those obtained in human patients with neglect (Fleet, Valenstein, Watson, \& Heilman, 1987; Geminiani, Bottini, \& Sterzi, 1998; Hurford, Stringer, \& Jann, 1998). Previous studies have revealed that recovery from AGm-induced neglect occurs in three contexts: (1) Spontaneous recovery occurs over the course of 3-8 weeks, although typically it is incomplete (Corwin 
et al., 1986; Crowne \& Pathria, 1982; Vargo, Corwin, King, \& Reep, 1988); (2) acute drug-induced recovery is produced by systemic injection of dopaminergic (DA) agonists (Corwin et al., 1986; King \& Corwin, 1990), and (3) accelerated recovery is produced by $48 \mathrm{~h}$ of light deprivation (Corwin \& Vargo, 1993; Crowne et al., 1983; Vargo, Lai, \& Marshall, 1998).

The results of a number of recent studies have suggested that the striatum may play a crucial role in neglect and recovery from neglect produced by AGm lesions. These studies indicate that alterations in striatal function are correlated with neglect and behavioral recovery (Vargo et al., 1998; Vargo \& Marshall, 1995, 1996a, 1996b). Vargo and Marshall (1996b) found that receptor binding to kainate and $N$-methyl-D-aspartate (NMDA) receptors was reduced in the ipsilateral dorsolateral quadrant of the striatum, relative to the contralateral striatum, in subjects with severe neglect. Kainate receptor levels returned to normal, and NMDA receptor levels increased in the ipsilateral dorsolateral striatum in a time frame correlated with spontaneous behavioral recovery. Furthermore, Vargo and Marshall $(1995,1996 a)$ found that levels of immediate early gene (IEG) expression in the dorsolateral quadrant of the ipsilateral striatum were reduced, relative to the contralateral striatum, in animals with neglect. Following spontaneous or light-deprivation-induced recovery, striatal IEG expression was symmetrical (Vargo et al., 1998; Vargo \& Marshall, 1995, 1996a).

Anatomical studies of the dorsolateral quadrant of the striatum reveal that this area encompasses the dorsocentral striatum (DCS), the projection zone of AGm efferents (Reep \& Corwin, 1999). The DCS receives glutamatergic (GLU) projections from the AGm (Corwin \& Reep, 1998; Divac, Fonnum, \& Storm-Mathisen, 1979; Reep et al., 1987) and DA projections from the substantia nigra (SN; Parent, 1990). These cortical GLU and subcortical DA inputs converge upon the same medium spiny neurons in the striatum (Alexander \& Crutcher, 1990; Parent, 1990). Alterations in both GLU and DA mechanisms are associated with AGm-lesion-induced neglect and recovery. Unilateral destruction of the AGm, which removes a large GLU input to the dorsolateral striatum, produces severe neglect (Corwin et al., 1991; Vargo \& Marshall, 1996b), and glutamate receptor changes in this region of the striatum are correlated with neglect and behavioral recovery (Vargo \& Marshall, 1996b). Also, systemic administration of the dopamine agonist apomorphine can produce dramatic acute recovery from neglect (Corwin et al., 1986).

The results of the metabolic and pharmacological studies suggest that neglect induced by lesions of the $\mathrm{AGm}$ may be caused by disruption of a corticostriatal circuit from the AGm to the dorsolateral striatum and that normalization of function within this region is correlated with behavioral recovery from neglect (Corwin et al., 1991; Vargo \& Marshall, 1996a, 1996b). However, these studies are correlational and have focused on the entire dorsolateral quadrant of the striatum. The anatomical findings indicate that a specific subzone within the dorsolateral striatum, the DCS, receives dense projections from the AGm (Reep \& Corwin, 1999; Reep et al., 1987), implicating this area as a potentially crucial site related to neglect and recovery. In order to examine this issue, in the present study, the effects of unilateral destruction of the DCS on responsiveness to visual, tactile, and auditory stimulation were examined. If the DCS is related to neglect and behavioral recovery following AGm lesions, it would be expected that a unilateral lesion of the DCS would produce multimodal neglect.

\section{METHOD}

\section{Subjects}

The subjects consisted of 23 male Long-Evans hooded rats bred from stock purchased from Harlan Sprague-Dawley. The animals were individually housed under standard laboratory conditions. Throughout the study, the rats were under a 12:12-h light:dark schedule, with food and water available ad lib. Prior to the surgical procedures, the subjects were handled daily (1-2 $\mathrm{min} /$ day) for 21-28 days, to gentle them for subsequent behavioral testing. The animals were tested within the later half of the 12-h light cycle between 1400 and $1700 \mathrm{~h}$.

\section{Surgical Procedure}

The animals were anesthetized with sodium pentobarbitol at a dosage of $50 \mathrm{mg} / \mathrm{kg}$ i.p. When totally unresponsive, as determined by the absence of corneal reflex and unresponsiveness to a mild tail pinch, the subjects were placed in a stereotaxic apparatus. Blunttipped ear bars were used to prevent damage to the tympanic membrane. The animals were randomly assigned to one of the following groups: (1) a DCS group $(n=7)$, which received NMDA at a concentration of $20 \mu \mathrm{g} / \mu \mathrm{l}$ in $0.1 \mathrm{M}$ phosphate-buffered saline (PBS); (2) a lateral striatal control (LSC) group $(n=7)$, which received NMDA at a concentration of $20 \mu \mathrm{g} / \mu \mathrm{l}$ in $0.1 \mathrm{M}$ PBS, chosen because this region receives projections from the lateral agranular cortex, a region not implicated in neglect (Crowne et al., 1983; Vargo et al., 1988); and (3) a vehicle (VEH) control group ( $n=9$ ), which received an injection of $0.1 \mathrm{M}$ PBS in the DCS. Under aseptic conditions following a Betadine scrub, a small hole of approximately $2.0 \mathrm{~mm}$ was drilled in the skull on the left side, at either $0.5 \mathrm{~mm}$ rostral and $3.4 \mathrm{~mm}$ lateral to bregma (DCS and VEH groups) or $0.5 \mathrm{~mm}$ rostral and $4.4 \mathrm{~mm}$ lateral to bregma (LSC group). The injection procedures were identical to those of Sananes and Davis (1992). The drugs were infused with a 26-gauge Hamilton syringe lowered $4.2 \mathrm{~mm}$ ventral to the dura for the DCS and VEH groups and $5.2 \mathrm{~mm}$ ventral for the LSC group. The $0.1-\mu$ dose of NMDA or PBS was infused at a rate of $0.1 \mu \mathrm{l} / 2 \mathrm{~min}$ and were allowed to diffuse for an additional 2 min before the syringe was removed. Following surgery, the incisions were sutured and treated with Neosporin ointment topically, and the rats were kept warm and were monitored prior to being returned to the colony.

\section{Behavioral Testing}

Testing for neglect began at $24 \mathrm{~h}$ postinjection. Behavioral testing was identical to that used in previous studies (e.g., Corwin et al., 1986), which is a modification of the testing procedure of Crowne and Pathria (1982). The animal was transferred in the home cage to a testing platform for a 1 -min period of adaptation. During habituation, the subject was observed for any consistent circling tendencies or motor asymmetries. The animal was then placed directly on a test platform, on which markings delineate $0^{\circ}, 30^{\circ}, 45^{\circ}$, and $60^{\circ}$ angles in either direction from a central line running the length of the testing board. The subject was aligned with the center line of the 
board and was gently restrained by hand from behind without restricting head movement. The stimuli were presented only when there was no evidence of struggling and no asymmetry of body posture and when the head was oriented in direct line with the body. Visual, tactile, and auditory stimuli were then presented in turn (order of stimulus presentation has been shown to have no effect on the results; Vargo et al., 1988). The visual stimuli consisted of the presentation of a silver metallic rod $10.0 \mathrm{~cm}$ in length, which was waved in small circles (approximately $5.0 \mathrm{~cm}$ in diameter) five times within the animal's visual field at a distance of $7.5-10.0 \mathrm{~cm}$ from the animal. The auditory stimulus was a single click, approximately 114-dB SPL, generated by a clicking device held at midbody. The tactile stimulus was a single caudal-to-rostral stroke through the vibrissae with a $15-\mathrm{cm}$ Puritan applicator (Harkwood Products Co., No. 807). Although there was a visual component to this stimulation, Corwin (unpublished data) found that rats orient to stimulation of the vibrissae under dim red-light conditions and do not respond under lighted conditions to the probe when the vibrissae are not contacted.

Three cycles made up one test session. One cycle consisted of a single presentation of each of the three stimuli to each side of the body. The experimenter rated the degree of head turn toward (appropriate responding) or away from (allesthetic/allokinetic responding) the stimuli, as measured by the position of the tip of the snout over the test platform markings. Head turns of less than $30^{\circ}$ were scored as 0 , turns between $30^{\circ}$ and $45^{\circ}$ as 1 , those between $45^{\circ}$ and $60^{\circ}$ as 1.5 , and those greater than $60^{\circ}$ as 2 . Orientations made more than $2 \mathrm{sec}$ after stimulus presentation received a score of 0 . Previous studies have found that the use of this scoring procedure produces an interrater reliability of 1.0 for the direction of orientation and greater than 0.90 reliability for the magnitude of orientation (Corwin \& Vargo, 1993; King \& Corwin, 1990; King et al., 1989; Vargo et al., 1988). Appropriate orientations and allesthetic/ allokinetic responses were scored separately. With this scoring method, the maximal score for appropriate responding was 6 for each of the three stimuli or 18 total for each side of the body. The subject had to achieve a score of 5 on either side of the body and had to respond in two stimulus modalities, in order to be considered responsive. If the criteria for responsiveness were not met, that testing session was not used in the statistical analysis. If the minimum level of responsiveness was not met for two tests in a particular week, that week was not included in the analyses. This occurred in only 2 subjects in the VEH group, each for 1 week of testing. During behavioral testing, the experimenter was blind with respect to group affiliation.

Two total orientation scores (one for each side of the body) were obtained for each test by summing all the scores representing appropriate responding. Thus, the total ipsilateral orientation score represents the sum of all the scores associated with appropriate responding to stimuli presented to the lesioned side of the body. Likewise, the total contralateral orientation score represents the sum of all the scores associated with appropriate responding to stimuli presented to the contralateral side of the body. A total neglect ratio was derived from the formula (total contralateral orientation score)/ (total ipsilateral orientation score). The neglect ratio takes into account total responsiveness and is a measure of relative responding to each side of the body; the ratio also indicates asymmetries in orientation behavior. Because the neglect ratio consists of the orientations in each of the modalities, individual modality neglect ratios were calculated with the formula (contralateral modality responsiveness ipsilateral modality responsiveness)/(contralateral modality responsiveness + ipsilateral modality responsiveness). This ratio was utilized because of the large number of zero scores obtained in the individual modalities. After the initial test for neglect, the animal was returned to the home cage and was monitored twice daily. Testing continued three times per week for the following 3 weeks.

\begin{abstract}
Histology
After behavioral testing was completed, the rats were sacrificed by an overdose of sodium pentobarbital $(65 \mathrm{mg})$ and, when totally unresponsive (absence of a corneal reflex and unresponsiveness to a mild tail pinch), were intracardially perfused with normal saline followed by $10 \%$ formalin. The brain was removed from the skull, placed in $10 \%$ formalin for at least 3 days, then frozen and sectioned in the coronal plane at $50 \mu \mathrm{m}$. To assess for lesion extent, alternate sections through the lesion were mounted and stained with cresyl violet. Lesion size was measured by tracing the extent of the lesion, using an image analysis software program (Optimas; BioScan, Edmonds, WA). All histology was done with the experimenter blind with respect to the subject's behavioral performance and group affiliation.
\end{abstract}

\section{RESULTS}

\section{Lesion Extent}

The total extents of the lesions for subjects in the DCS and LSC groups were traced onto standard brain diagrams (Paxinos \& Watson, 1998) and were measured, using an image analysis program (Optimas; BioScan, Edmonds, WA). Figure 1 illustrates the individual subjects in each of the groups that represent the maximum and the minimum lesion extents. With the exception of minute cannula tract damage in $30 \%$ of the subjects (the lateral agranular cortex in the DCS group and the forelimb/parietal cortex 1 in the LSC group), lesions in the DCS and LSC groups were confined to the striatum-the largest lesion extending caudally $1.26 \mathrm{~mm}$, beginning at the level of the crossing of the anterior commissure. The cannula tract did not infringe upon the AGm in any of the subjects. Infusion of NMDA produced a teardrop-shaped lesion confined to the cells within the DCS and LSC areas. Fiber fascicles piercing the striatum were still present in the DCS and LSC areas. Damage to the DCS was evidenced by an absence of cell bodies in the area designated as receiving cortical projections from the AGm (Reep et al., 1987). Comparable destruction of the LSC area, located just ventral and lateral to the DCS, was also evidenced by the absence of cell bodies. A one-way group $\times$ total lesion extent analysis of variance (ANOVA) revealed no significant difference in the amount of striatal damage between the LSC and the DCS groups $[F(1,12)=1.856, p=.198]$, indicating that destruction of striatal cells was comparable in both manipulation groups.

\section{Total Neglect Ratios}

Mean total neglect ratios were analyzed for each of the 3 weeks. Figure 2 illustrates the total neglect ratios for each week for each of the groups. In order to examine whether a significant difference in total neglect ratios existed between or within groups as a function of time, a mixed group $\times$ weeks ANOVA was performed. The results indicated a significant main effect only of group $[F(2,19)=$ $13.668, p<.0009]$. A post hoc Student Newman-Keuls (SNK) analysis of mean total neglect ratios, collapsed across weeks, revealed that the DCS group differed significantly from the LSC and VEH groups ( $p$ s $<.05$ ), in- 

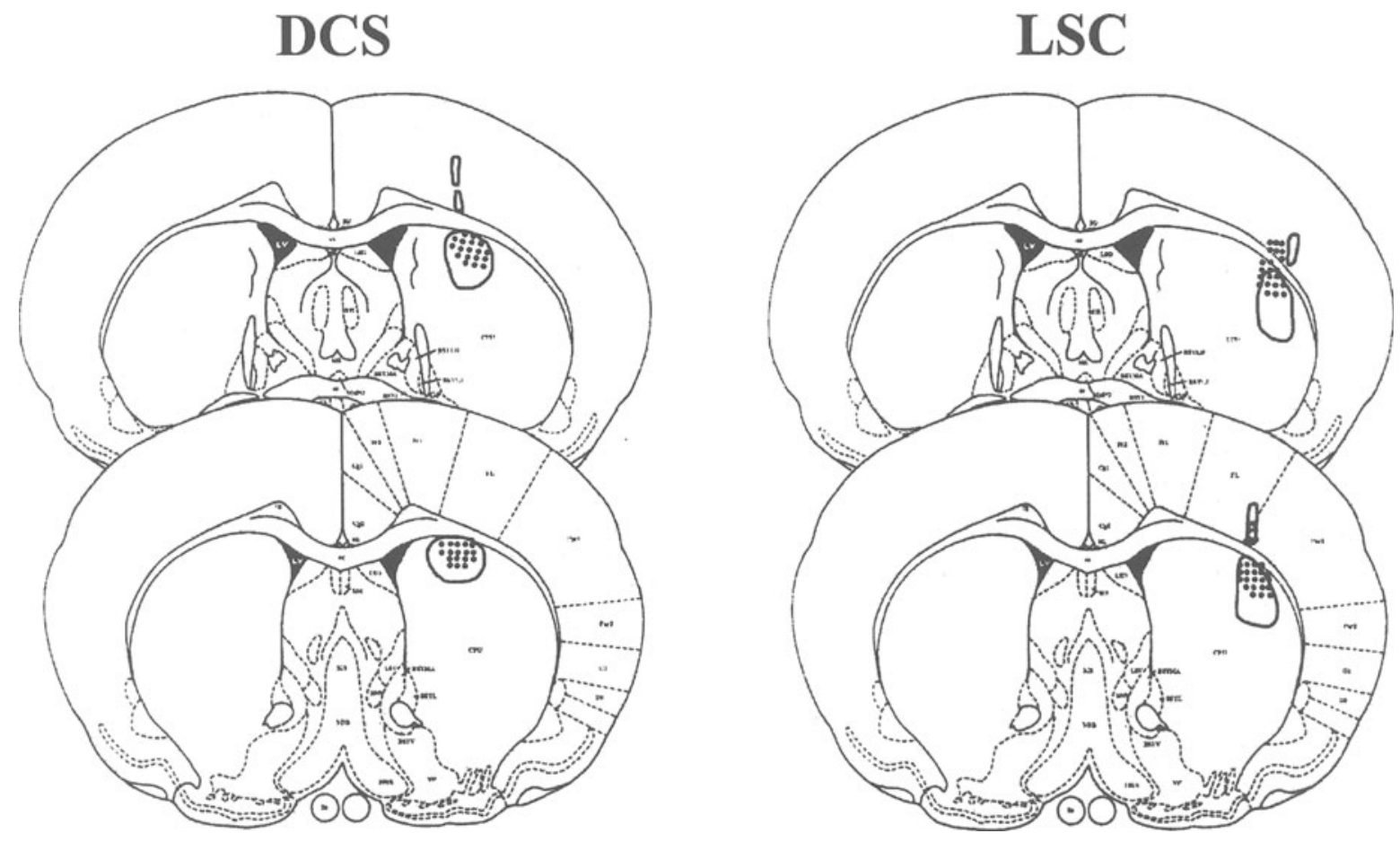

Figure 1. Maximum (outline) and minimum (stipple) extents of the lesion for individual subjects in the dorsocentral striatum (DCS) and lateral striatal control (LSC) groups.

dicating that the DCS group demonstrated more severe neglect than did the other groups. The LSC group did not differ from the VEH group. The lack of a significant interaction suggests that the animals demonstrated no behavioral recovery over the 3 weeks of testing. Also, observations of motor behavior during the $1 \mathrm{~min}$ of habituation prior to behavioral testing revealed that the differences in orientation to the ipsilateral and the contralateral sides are not explainable by any obvious changes in circling behavior or by motor asymmetry.

Three of the DCS operates were tested at times ranging from 60 to 96 days postsurgery, in order to assess spontaneous recovery, because there was no evidence of spontaneous recovery in the DCS operates at the end of the 3 rd week of testing (see Figure 2). The results of the behavioral tests at 60-96 days postsurgery indicated that these subjects still had severe neglect (mean total neglect ratio $=0.10$ ) and evidenced no sign of spontaneous recovery.

\section{Raw Scores}

Because changes in the neglect ratios may be caused by changes in responsiveness on the contralateral side, the ipsilateral side, or both, separate analyses of the contralateral and ipsilateral raw score data were also performed. A group $\times$ weeks ANOVA was conducted on the raw score means of contralateral and ipsilateral responding. Figure 3 illustrates the mean contralateral and ipsilateral responsiveness for each group, collapsed across the 3 weeks of testing. The ipsilateral and contralateral raw scores for each of the weeks are presented in Table 1.

Contralateral responsiveness. A group $\times$ weeks ANOVA revealed significant main effects of group $[F(2,19)=8.443, p<.002]$ and week $[F(1,19)=19.141$, $p<.0009]$. A post hoc SNK analysis performed on mean contralateral responsiveness, collapsed across weeks, revealed that the DCS group exhibited significantly less total contralateral responding than did the LSC and VEH groups $(p s<.05)$. The LSC and the VEH groups did not differ in the amount of contralateral responsiveness. A post hoc SNK analysis of contralateral responsiveness, collapsed across groups, revealed that the subjects demonstrated significantly more contralateral responding in the 1 st week of testing than in the 3 rd week, perhaps because the animals were unaccustomed to the stimuli and responded with more vigor in the 1 st week or because there was a deterioration in ability to respond in the 3rd week.

Ipsilateral responsiveness. A group $\times$ weeks ANOVA revealed a significant effect only of week $[F(1,19)=$ $18.252, p<.0009]$ ]. These results indicate that none of the manipulations created a group difference in ipsilateral responsiveness. However, a post hoc SNK analysis of ipsilateral responsiveness, collapsed across groups, revealed that the subjects, regardless of group affiliation, responded more during the lst week of testing than during the other weeks. Again, this may have occurred because the animals were unaccustomed to the stimuli and responded with more vigor in the 1 st week or because there 


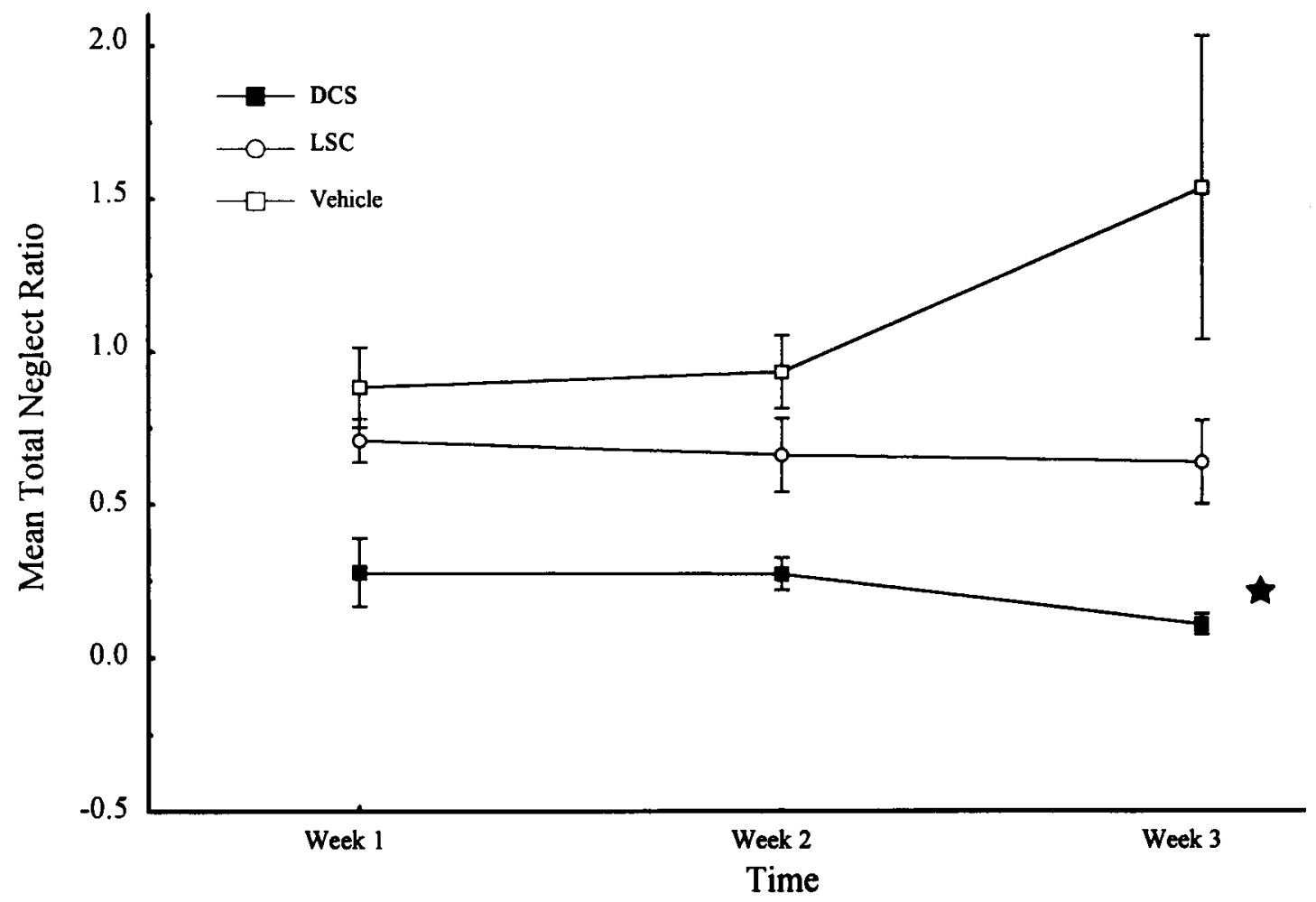

Figure 2. Total neglect ratios in the dorsocentral striatum (DCS), lateral striatal control (LSC), and vehicle control groups as a function of weeks. A ratio of 1.0 represents symmetrical responding. $A$ * indicates a significant difference from all of the other groups $(p<.05)$. Error bars represent standard errors.

was a deterioration in ability to respond in subsequent weeks $(p \mathrm{~s}<.05)$. There was no significant difference in ipsilateral responding between the second and the third weeks.

\section{Modalities}

Neglect ratios within separate modalities were analyzed, in order to examine whether there were differences between or within groups in orientation in each of the modalities over time. Figure 4 illustrates the mean modality neglect ratios for each group across the 3 weeks of testing.

Visual. In the visual modality, the group $\times$ weeks ANOVA revealed a significant main effect only of group $[F(2,19)=12.494, p<.0009]$. A post hoc SNK analysis of mean visual neglect ratios, collapsed across weeks, revealed that the DCS group differed significantly from the LSC and VEH groups ( $p \mathrm{~s}<.05$ ), indicating that the DCS group exhibited significantly greater contralateral neglect of visual stimulation than did the other groups. The LSC and VEH groups did not differ in the severity of visual neglect.

Tactile. In the tactile modality, the group $\times$ weeks ANOVA revealed a significant main effect only of group $[F(2,19)=6.930, p<.005]$. A post hoc SNK analysis of mean visual neglect ratios, collapsed across weeks, revealed that the DCS group differed significantly from the LSC and VEH groups ( $p s<.05)$, indicating that the DCS group exhibited significantly greater neglect of tactile stimulation than did the other groups. The LSC and the VEH groups did not differ in tactile neglect ratios.

Auditory. In the auditory modality, the group $x$ weeks ANOVA revealed no significant differences. The failure to find significant auditory neglect is not uncommon, even following AGm lesions (Corwin \& Vargo, 1993), and may be due to some variability in the presentation of neglect symptoms within the individual modalities.

\section{Allesthesia/Allokinesia}

A series of Wilcoxon matched-pairs signed-ranks tests was done comparing the summed ipsilateral and contralateral allesthetic/allokinetic responses for each of the groups. The results revealed no significant differences in allesthesia/allokinesia between the two sides of the body for any of the groups. The failure to find significant allesthesia is not surprising, considering that, in AGm operates, significant allesthesia/allokinesia is only found following very large lesions that destroy the entire rostral AGm.

\section{DISCUSSION}

The results of the present study indicate that selective unilateral destruction of the DCS produces severe mul- 


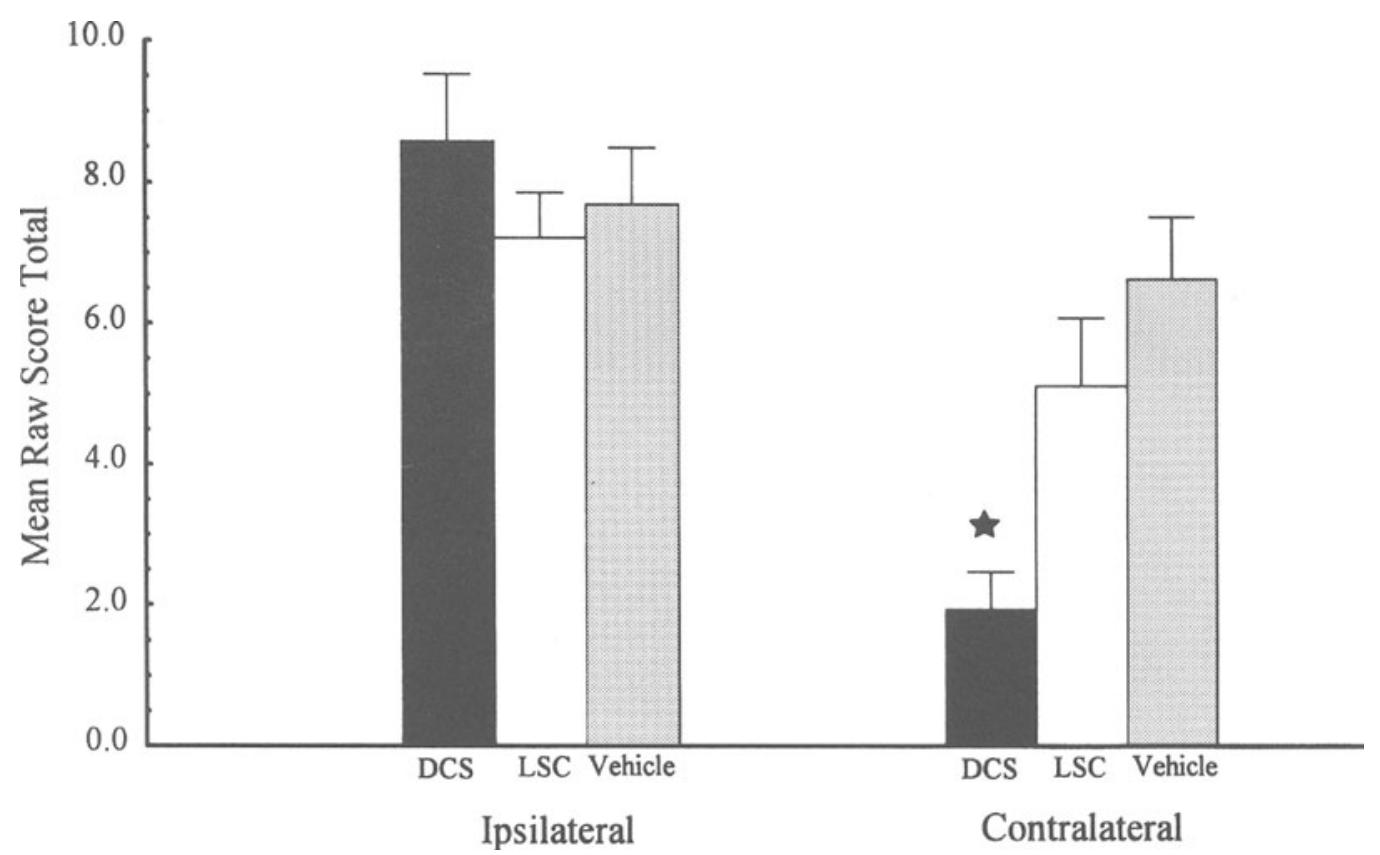

Figure 3. Total ipsilateral and contralateral responsiveness for the dorsocentral striatum (DCS), lateral striatal control (LSC), and vehicle control groups, collapsed across weeks. A score of 18.0 represents complete responsiveness across all modalities. $A$ * indicates a significant difference from all of the other groups $(p<.05)$. Error bars represent standard errors.

timodal neglect. LSC rats with comparable lesions of the adjacent region of the striatum or VEH controls did not demonstrate severe neglect. These results support the findings of prior studies that have implicated the dorsolateral striatum in neglect and recovery from neglect (Corwin et al., 1991; Vargo et al., 1998; Vargo \& Marshall 1995, 1996a, 1996b) and extend those findings by indicating that the crucial site in the dorsolateral striatum may be the DCS. In addition, the present study is supported by the findings of Marshall, Berrios, and Sawyer (1980), which indicated that there are regional differences in the behavioral effects of DA depletion in the striatum.

Anatomical studies have found that the AGm projects to the DCS (Berendse \& Groenewegen, 1990; Reep \& Corwin, 1999; Reep et al., 1987) and that these areas, together with associated thalamic nuclei, may form a network underlying neglect and spatial processing (Groenewegen, 1988 ), as has been proposed for primates (Alexander, DeLong, \& Strick, 1986; Mesulam, 1990). Unilateral destruction of the AGm produces severe multimodal neglect (Corwin et al., 1986; King et al., 1989). Likewise, unilateral damage to the DCS produces asymmetries in responsiveness that are qualitatively and quantitatively similar to those produced by destruction of the AGm, supporting the hypothesis that these areas function as a system. The present findings are quite comparable with those from a number of studies that address the similar effects of lesions of specific prefrontal cortical regions and their projection regions in the striatum (Divac, Markow- itsch, \& Pritzel, 1978; Dunnett \& Iversen, 1980, 1981; Pisa, 1988).

The importance of the striatum in AGm-lesioninduced neglect may help to provide an explanation for pharmacological studies that indicate that GLU and DA mechanisms may be involved in recovery. The results of anatomical studies indicate that there are parallel circuits involving the cortex, the basal ganglia, and the thalamus (Alexander et al., 1986; Groenewegen, 1988). Generally, these circuits consist of a specific cortical area that sends excitatory GLU projections (Divac et al., 1979) to medium spiny neurons in a selected region of the striatum, which, in turn, project via two distinct pathways to the SN pars reticulata (Alexander \& Crutcher, 1990). These medium spiny neurons also receive DA projections from the $\mathrm{SN}$ pars compacta. Within specific subregions of the striatum, cortical GLU projections converge on the same medium spiny neurons as do subcortical DA projections. It has been suggested that cortical lesions that decrease GLU input to the SN may also produce a decrease in striatal extracellular DA (Emmi, Rajabi, \& Stewart, 1996). Thus, cortical lesions may affect both DA and GLU function in a specific region of the striatum. A number of behavioral studies have found that administration of DA agonists produces dramatic behavioral recovery from neglect induced by unilateral cortical or SN lesions in rats (Corwin, Burcham, \& Hix, 1996; Corwin et al., 1986; Marshall, 1979; Marshall \& Gotthelf, 1979) and by cortical lesions in humans (Fleet et al., 1987; Geminiani et al., 1998; Hurford et al., 
Table 1

Contralateral and Ipsilateral Responsiveness Across Modalities: Group Means and

Standard Errors Across 3 Weeks of Testing

\begin{tabular}{|c|c|c|c|c|c|c|}
\hline \multirow[b]{2}{*}{ Group } & \multicolumn{2}{|c|}{ Week 1} & \multicolumn{2}{|c|}{ Week 2} & \multicolumn{2}{|c|}{ Week 3} \\
\hline & $M$ & $S E$ & $M$ & $S E$ & $M$ & $S E$ \\
\hline \multicolumn{7}{|c|}{ Contralateral } \\
\hline DCS & $2.79^{\circ}$ & \pm 2.45 & 2.19 & \pm 1.23 & 0.85 & $\pm 0.85^{*}$ \\
\hline LSC & 7.02 & \pm 2.80 & 4.55 & \pm 2.80 & 3.79 & \pm 2.89 \\
\hline Saline & 7.63 & \pm 3.37 & 6.58 & \pm 2.65 & 5.71 & \pm 2.75 \\
\hline \multicolumn{7}{|l|}{ Ipsilateral } \\
\hline DCS & 9.69 & \pm 3.97 & 7.86 & \pm 2.36 & 8.21 & \pm 2.01 \\
\hline LSC & 9.74 & \pm 3.06 & 6.29 & \pm 1.69 & 5.64 & \pm 1.55 \\
\hline Saline & 9.50 & \pm 3.18 & 7.35 & \pm 2.12 & 6.25 & \pm 2.97 \\
\hline
\end{tabular}

Note-DCS, dorsolateral striatum; LSC, lateral striatal control. ${ }^{*} p<.05$.

1998). Furthermore, DA receptor antagonists reinstate neglect after spontaneous recovery (Corwin et al., 1986; Vargo, Bromberg, Best, Corwin, \& Marshall, 1996; Vargo, Richard-Smith, \& Corwin, 1989). These results suggest that manipulation of DA mechanisms may compensate for disruptions in cortical input (Corwin et al., 1996; Corwin et al., 1986) and also support the hypothesis that the effects of DA agonists and antagonists on neglect induced by AGm lesions may be produced by their actions on the dorsolateral striatum, perhaps in the DCS.

The role of the DCS in neglect and behavioral recovery is further supported by recent findings from our laboratory. If the DCS is the site of action of apomorphine, removal of this area would prohibit recovery. Recent findings in several pilot subjects support this hypothesis. Rats with unilateral DCS lesions did not demonstrate behavioral recovery following administration of $0.5 \mathrm{mg} / \mathrm{kg}$ of apomorphine, the most effective therapeutic dosage from prior studies of AGm- (Corwin et al., 1986) and PPC-lesion-induced neglect (Corwin et al., 1996). Furthermore, we found that direct injection of apomorphine into the DCS produced dramatic acute recovery from severe neglect induced by unilateral AGm lesions, whereas injections of apomorphine to the laterally adjacent striatum (an LSC group) did not produce recovery (Van Vleet, Corwin, \& Burcham, 1999).

The results of these studies support the findings of Vargo and Marshall (1995, 1996a, 1996b) regarding the important role of the dorsolateral striatum in AGminduced neglect. They found that changes in GLU receptor levels and IEG expression in the dorsolateral quadrant of the striatum were correlated with spontaneous behavioral recovery from $\mathrm{AGm}$-lesion-induced neglect (Vargo \& Marshall, 1995, 1996a, 1996b). In the present study, the animals with DCS lesions were tested for at least 3 weeks postsurgery and demonstrated no spontaneous behavioral recovery ( 3 DCS subjects were tested for neglect for up to 96 days postsurgery, and all exhibited severe neglect). The lack of recovery in the present study provides further evidence that an intact DCS may be necessary for spontaneous recovery to occur. In addition, light-deprivation-induced accelerated recovery from se- vere neglect produced by $\mathrm{AGm}$ lesions is correlated with symmetrical IEG expression in the dorsolateral striatum (Vargo et al., 1998). These studies suggest that some compensatory changes correlated with recovery from unilateral AGm lesions are occurring in or near the DCS and that the DCS may maintain an integrative role of cortical GLU and subcortical DA projections that is necessary for behavioral recovery from neglect.

Studies in other species have suggested that the striatum is involved in sensory gating, attention, and sensorimotor integration (Krauthamer, 1979; Schneider, Diamond, \& Markham, 1987; Tatton, Eastman, Bedingham, Verrier, \& Bruce, 1984). Single-unit recording studies in both cats and monkeys have demonstrated that neurons in the caudate, the putamen, and the globus pallidus are involved in the evaluation of the behavioral significance of stimuli and the subsequent initiation of the appropriate motor action (Krauthamer, 1979; Schneider et al., 1987; Tatton et al., 1984). According to Gerfen (1992), converging GLU and DA inputs on medium spiny neurons in the striatum play a crucial role in motor control by disinhibiting the thalamus, thereby gating or facilitating cortically initiated movement. The results of these studies, together with the present findings, suggest that the DCS is involved in directed orientation to sensory stimulation in rodents.

However, two alternative explanations for the results in the present study must be considered. First, the apparent neglect found in the present study might be explainable by a deficit in response initiation to the side contralateral to the lesion. Data from two recent studies suggest that the asymmetries in responsiveness observed following DA depletion in the striatum reflect a deficit in motor output (response initiation), and not an impairment in attention (Brown \& Robbins, 1989; Ward \& Brown, 1996). Brown and Robbins found that rats with neglect induced by widespread unilateral 6-OHDA depletion of DA in the striatum exhibited a significant bias in responding toward the side of the lesion. Rats with neglect were also slower to initiate but not to complete contralateral responses, suggesting that the animals did not exhibit sensory loss or inattention but, rather, a deficit in motor output or response initiation (hemiakinesia). Ward and Brown examined the effects of striatal DA depletion in rats that were extensively pretrained to orient to a peripheral visual target, which was preceded by a visual cue that either directed or misdirected the animal to the subsequent target (covert orienting). DA depletion produced increased mean contralateral reaction times, and reaction times did not change differentially as a function of the attentional requirements of the task. Their results suggest that the deficits reflected a motor impairment, rather than an impairment in directing attention. The results of these studies suggest that a motor interpretation must be considered as an alternative explanation for the findings of the present study. However, there are a number of factors that mitigate the response initiation interpretation. In the Ward and Brown and the Brown and Robbins studies, the response initiation delay ranged from 100 

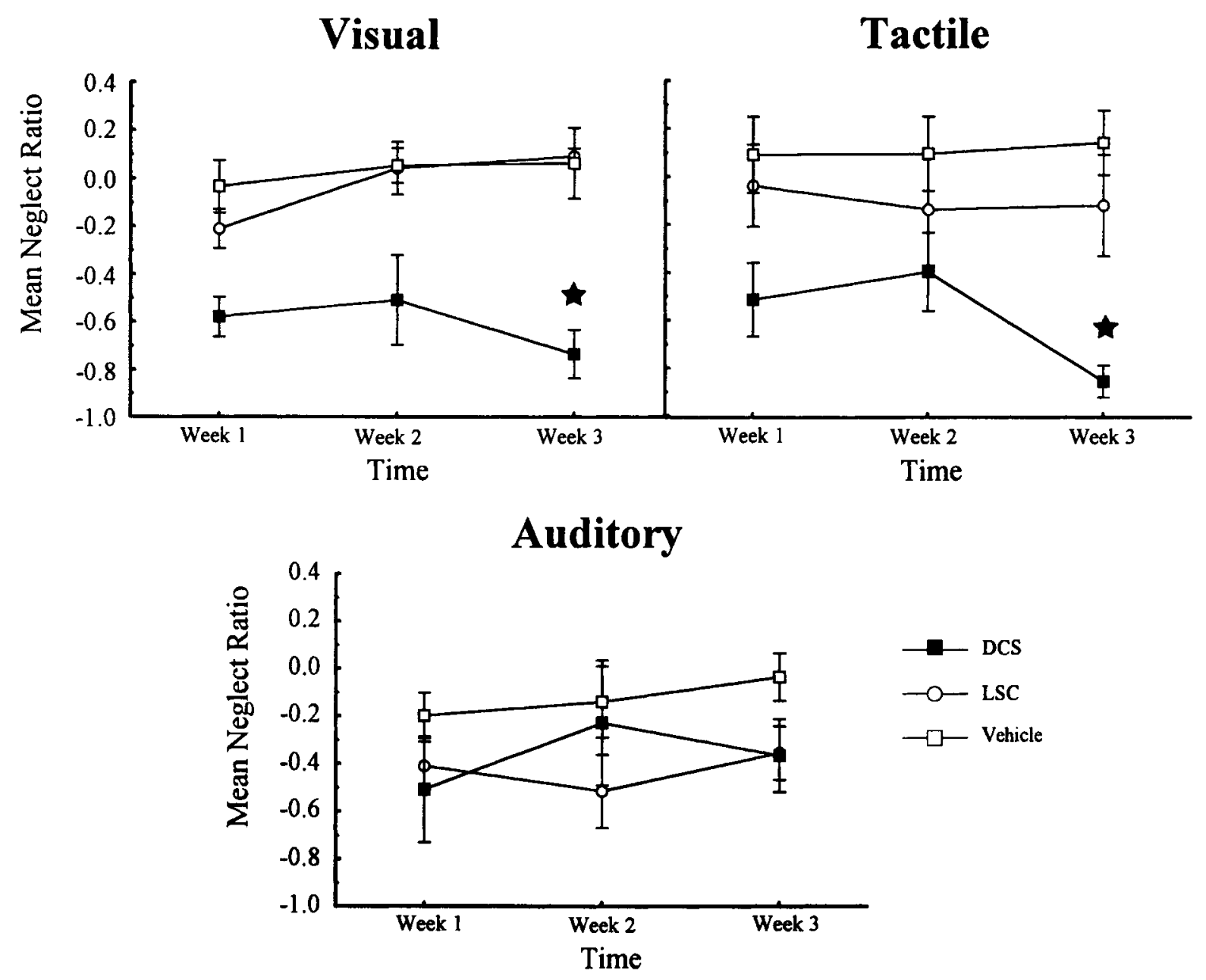

Figure 4. Individual modality neglect ratios in the dorsocentral striatum (DCS), lateral striatal control (LSC), and vehicle control groups as a function of weeks. A ratio of 0.0 represents symmetrical responding. $A$ * indicates a significant difference from all of the other groups $(p<.05)$. Error bars represent standard errors.

to $600 \mathrm{msec}$, respectively. Those animals with neglect in the present study frequently demonstrated a complete lack of contralateral responding to stimuli across the visual and tactile modalities, even though the subjects had a minimum of $2 \mathrm{sec}$ to respond and, in the case of visual stimulation, more than $5 \mathrm{sec}$ in which to respond. In addition, the level of DA depletion in the Brown and Robbins and the Ward and Brown studies was significant, whereas in the present study, given the small NMDA-induced lesions, it is unlikely that the level of DA depletion was extensive. Furthermore, there is no reason to expect that the level of depletion following DCS lesions, which induced severe neglect, should differ from that produced by virtually equivalent-sized lesions in the LSC group that did not exhibit neglect. Therefore, the results of the present study do not support a motor deficit interpretation (response initiation).

A second alternative explanation for the present findings might be that the DCS lesions resulted in an enhanced tendency to respond to the side ipsilateral to the lesion.
However, the behavioral findings do not support this interpretation. There were no differences among the groups in orienting to the ipsilateral side, such as might be expected if there was a dramatic tendency to turn toward the ipsilateral side in one of the groups. Furthermore, there were no differences among the groups in the tendency to respond to stimulation of the neglected side by orienting to the nonneglected side (allesthesia/allokinesia). Finally, during behavioral testing, there were no obvious changes in motor behavior (e.g.,turning behavior) that would lead to differences in responsiveness between the DCS and the LSC groups. Thus, it would seem that the neglect found in the present study resulted from a lack of responsiveness to stimulation of the neglected (contralateral) side and was not the result of enhanced turning to the ipsilateral side owing to motor impairment, inappropriate orientations, or differences in lesion size. Additional studies that involve testing measures that are specifically targeted at the potentially separable components of attention, sensory processing, and motor initiation in neglect are needed. 
Future investigations are needed to further examine the pharmacology and functional organization of the corticosubcortical circuitry addressed in the present study. Such investigations may include pharmacological manipulation of the DCS, physiological examinations of light-deprivationinduced recovery, and more focused anatomical studies. Drug infusion and physiological analyses of the DCS during light deprivation may provide insight into the mechanisms involved in the profound recovery obtained following this environmental manipulation. Anatomical studies may be useful in determining whether intact portions of the circuit for directed attention are involved in compensatory changes that may affect behavior following such lesions. Finally, the segregation of inputs from the cortex and the $\mathrm{SN}$ to either the striatal patch or the matrix may indicate another level of functional compartmental organization requiring investigation (Gerfen, 1992).

\section{REFERENCES}

Alexander, G. E., \& Crutcher, M. D. (1990). Functional architecture of basal ganglia circuits: Neural substrates of parallel processing. Trends in Neuroscience, 13, 241-247.

Alexander, G. E., De Long, M. R., \& Strick, P. L. (1986). Parallel organization of functionally segregated circuits linking basal ganglia and cortex. Annual Review of Neuroscience, 9, 357-381.

BERENDSE, H. W., \& GROENEWEGEN, H. J. (1990). Organization of the thalamostriatal projections in the rat, with special emphasis on the ventral striatum. Journal of Comparative Neurology, 299, 187-228.

Brown, V. J., \& Robbins, T. W. (1989). Deficits in response space following unilateral striatal dopamine depletion in the rat. Journal of Neuroscience, 9, 983-989.

Burcham, K. B., Corwin, J. V., Stoll, M. L., \& Reep, R. L. (1997) Disconnection of medial agranular and posterior parietal cortex produces multimodal neglect in rats. Behavioural Brain Research, 86, $41-47$.

Chander, H. C., King, V., Corwin, J. V., \& Reep, R. L. (1992). Thalamocortical connections of rat posterior parietal cortex in rats. Neuroscience Letters, 143, 237-242.

Corwin, J. V., Burcham, K. J., \& Hix, G. I. (1996). Apomorphine produces acute dose-dependent therapeutic effects on neglect produced by unilateral destruction of the posterior parietal cortex in rats. Behavioural Brain Research, 79, 41-49.

Corwin, J. V., Kanter, S., Watson, R. T., Hellman, K. M., ValenSTEIN, E., \& HASHIMOTO, A. (1986). Apomorphine has a therapeutic effect on neglect produced by unilateral dorsomedial prefrontal cortex lesions in rats. Experimental Neurology, 94, 683-689.

Corwin, J. V., King, V., Paulson, D., Watson, R. T., Heilman, K., \& REEP, R. L. (1991). Functional correlates of neglect produced by unilateral lesions of medial agranular cortex in the rat. Society for Neuroscience Abstracts, 17, 1584.

CoRwin, J. V., \& ReEP, R. L. (1998). Rodent posterior parietal cortex as a component of a cortical network mediating directed spatial attention. Psychobiology, 26, 87-102.

CoRwin, J. V., \& VARGO, J. M. (1993). Light deprivation produces accelerated behavioral recovery of function from neglect produced by unilateral medial agranular prefrontal cortex lesions in rats. Behavioural Brain Research, 56, 187-196.

Crowne, D. P., \& Pathria, M. N. (1982). Some attentional effects of unilateral frontal lesions in the rat. Behavioural Brain Research, 6 , 25-32.

Crowne, D. P., Richardson, C. M., \& Ward, G. (1983). Brief deprivation of vision after unilateral lesions of the frontal eye fields prevents contralateral inattention. Science, 220, 527-530.

Divac, I., Fonnum, F., \& Storm-Mathisen, J. (1979). High affinity uptake of glutamate in terminals of corticostriatal axons. Nature, 266, 377-378.
Divac, I., Markowitsch, H. J., \& Pritzel, M. (1978). Behavioral and anatomical consequences of small intrastriatal injections of kainic acid in the rat. Brain Research, 151, 523-532.

DunNetT, S. B., \& Iversen, S. D. (1980). Regulatory impairments following selective kainic acid lesions of the neostriatum. Behavioural Brain Research, 1, 497-506.

DunNetT, S. B., \& IVERSEN, S. D. (1981). Learning impairments following selective kainic acid-induced lesions within the neostriatum of rats. Behavioural Brain Research, 2, 189-209.

Emmi, A., Rajabi, H., \& Stewart, J. (1996). Behavioral and neurochemical recovery from partial 6-hydroxydopamine lesions of the substantia nigra is blocked by daily treatment with glutamate receptor antagonists MK-801 and CPP. Journal of Neuroscience, 16, 52165224.

Fleet, W. S., Valenstein, E., Watson, R. T., \& Heilman, K. M. (1987). Dopamine agonist therapy for neglect in humans. Neurology, $37,1765-1770$

Geminiani, G., Bottini, G., \& Sterzi, R. (1998). Dopamine stimulation in unilateral neglect. Journal of Neurosurgery \& Psychiatry, 65 , 345-347.

Gerfen, C. R. (1992). The neostriatal mosaic: Multiple levels of compartmental organization. Trends in Neuroscience, 15, 133-139.

GROENEWEGEN, H. J. (1988). Organization of the afferent connections of the mediodorsal thalamic nucleus in the rat related to the mediodorsalprefrontal topography. Neuroscience, 24, 379-431.

Heilman, K. M., Watson, R. T., \& Valenstein, E. (1993). Neglect and related disorders. In K. M. Heilman \& E. Valenstein (Eds.), Clinical Neuropsychology (3rd ed., pp. 243-293). New York: Oxford University Press.

Hurford, P., Stringer, A.Y., \& JANN, B. (1998). Neuropharmacologic treatment of hemineglect: A case report comparing bromocriptine and methylphenidate. Archives of Physical \& Medical Rehabilitation, 79, 346-349.

KING, V., \& Corwin, J. V. (1990). Neglect following unilateral ablation of the caudal but not the rostral portion of medial agranular cortex of the rat and the therapeutic effect of apomorphine. Behavioural Brain Research, 37, 169-184.

KING, V., \& CoRwin, J. V. (1992). Spatial deficits and hemispheric asymmetries in the rat following unilateral and bilateral lesions of the posterior parietal or medial agranular cortex. Behavioural Brain Research, 50, 53-68.

KING, V., \& CoRwIN, J. V. (1993), Comparisons of hemi-inattention produced by unilateral lesions of the posterior parietal cortex or medial agranular prefrontal cortex in rats: Neglect, extinction, and the role of stimulus distance. Behavioural Brain Research, 54, 117-131.

King, V., CoRwin, J. V., \& ReEP, R. L. (1989). Production and characterization of neglect in rats with unilateral lesions of ventrolateral orbital cortex. Experimental Neurology, 105, 289-299.

Krauthamer, G. (1979). Sensory functions of the neostriatum. In I. Divac (Ed.), The neostriatum (pp. 263-289). Oxford: Pergamon Press.

MarShall, J. F. (1979). Somatosensory inattention after dopaminedepleting intracerebral 6-OHDA injections: Spontaneous recovery and pharmacological control. Brain Research, 23, 127-145.

Marshall, J. F., Berrios, N., \& SAWYeR, S. (1980). Neostriatal dopamine and sensory inattention. Journal of Comparative \& Physiological Psychology, 94, 833-846.

Marshall, J. F., \& GotThelf, T. (1979). Sensory inattention in rats with 6-hydroxydopamine-induced degeneration of ascending dopaminergic neurons: Apomorphine-induced reversal of deficits. Experimental Neurology, 65, 398-411.

Mesulam, M. M. (1990). Large-scale neurocognitive networks and distributed processing for attention, language, and memory. Annals of Neurology, 28, 597-613.

Parent, A. (1990). New frontiers in basal ganglia research. Trends in Neuroscience, 13, 241-244.

Paxinos, B., \& Watson, C. (1998). The rat brain in stereotaxic coordinates (2nd ed.). New York: Academic Press.

PISA, M. (1988). Motor somatotopy in the striatum of the rat: Manipulation, biting and gait. Behavioural Brain Research, 27, 21-35.

Reep, R. L., Chandler, H. C., \& Corwin, J. V. (1994). Rat posterior 
parietal cortex: Topography of corticocortical and thalamic connections. Experimental Brain Research, 100, 67-84.

REEP, R. L., \& CoRwIN, J. V. (1999). Topographic organization of the striatal and thalamic connections of rat medial agranular cortex. Brain Research, 841, 43-52.

Reep, R. L., Corwin, J. V., Hashimoto, A., \& Watson, R. T. (1984). Afferent connections of medial prefrontal cortex in rats. Neuroscience Letters, 44, 247-252.

ReEP, R. L., CoRWin, J. V., Hashimoto, A., \& Watson, R. T. (1987) Efferent connections of the rostral portion of medial agranular cortex in rats. Brain Research Bulletin, 19, 203-221.

ReEP, R. L., Goodwin, G. S., \& CoRwiN, J. V. (1990). Topographic organization in the corticocortical connections of medial agranular cortex in rats. Journal of Comparative Neurology, 294, 149-163.

SANANES, C. B., \& Davis, M. (1992). $N$-methyl-D-aspartate lesions of the lateral and basolateral nuclei of the amygdaloid block fear-potentiated startle and shock sensitization of startle. Behavioral Neuroscience, 106, $72-80$.

Schneider, J. S., Diamond, S. D., \& Markham, C. H. (1987). Parkinson's disease: Sensory and motor problems in arms and hands. $\mathrm{Neu}$ rology, 37, 951- 956.

Tatton, W. G., Eastman, M. J., Bedingham, W., Verrier, M. C., \& BRuCE, I. C. (1984). Defective utilization of sensory input as the basis for bradykinesia, rigidity and decreased movement repertoire in Parkinson's disease: A hypothesis. Canadian Journal of Neurological Science, 11(Suppl.), 136-143.

Vallar, G., \& Perani, D. (1986). The anatomy of unilateral neglect after right-hemisphere stroke lesions: A clinical/CT-scan correlation study in man. Neuropsychologia, 24, 609-622.

VAN VleET, T. M., Corwin, J. V., \& BurchaM, K. B. (1999). Infusion of apomorphine into the dorsal central striatum produces acute recovery from AGm-induced neglect in rats. Society for Neuroscience Abstracts, 25, 1896.
VARgo, J. M., Bromberg, B. B., Best, P. J., CoRwin, J. V., \& MarSHALL, J. F. (1996). D1-class dopamine receptor involvement in the behavioral recovery from prefrontal cortical injury. Behavioural Brain Research, 72, 39-48.

VARGo, J. M., CORWIN, J. V., KING, V., \& REEP, R. L. (1988). Hemispheric asymmetry in neglect produced by unilateral lesions of dorsomedial prefrontal cortex in rats. Experimental Neurology, 102, 199-209.

VARGo, J. M., LAI, H. V., \& MARShaLl, J. F. (1998). Light deprivation accelerates recovery from frontal cortical neglect: Relation to locomotion and striatal fos expression. Behavioral Neuroscience, 112, 387-398.

Vargo, J. M., \& Marshall, J. F. (1995). Time-dependent changes in dopamine agonist-induced striatal fos immunoreactivity are related to sensory neglect and its recovery after unilateral prefrontal cortex injury. Synapse, 20, 305-315.

VARGo, J. M., \& MARShaLL, J. F. (1996a). Frontal cortex ablation reversibly decreases striatal zif/268 and junB expression: Temporal correspondence with sensory neglect and its spontaneous recovery. Synapse, 22, 291-303.

VARGO, J. M., \& MARSHALL, J. F. (1996b). Unilateral frontal cortex ablation producing neglect causes time-dependent changes in striatal glutamate receptors. Behavioural Brain Research, 77, 189-199.

VARGo, J. M., RichaRd-Smith, M., \& CoRwIN, J. V. (1989). Spiroperidol reinstates asymmetries in neglect in rats recovered from left vs. right dorsomedial prefrontal cortex lesions. Behavioral Neuroscience, 103, 1017-1027.

WARD, N. M., \& BRowN, V. J. (1996). Covert orienting of attention in the rat and the role of striatal dopamine. Journal of Neuroscience, 16, 3082-3088.

(Manuscript received July 8, 1999;

revision accepted for publication September 23,1999 .) 